\title{
Existence of solutions for G-SDEs with upper and lower solutions in the reverse order
}

\author{
Faiz Faizullah ${ }^{1 *}$ and Daxiong Piao ${ }^{2}$ \\ ${ }^{1}$ College of Physical and Environmental Oceanography, Ocean University of China, Qingdao 266100, PR China. \\ ${ }^{2}$ School of Mathematical Sciences, Ocean University of China, Qingdao 266100, PR China.
}

Accepted 16 December, 2011

\begin{abstract}
The existence of solutions for stochastic differential equations under G-Brownian motion (G-SDEs) of the type $X_{t}=X_{0}+\int_{0}^{t} b\left(v, X_{v}\right) d v+\int_{0}^{t} \theta\left(v, X_{v}\right) d\langle B\rangle_{v}+\int_{0}^{t} \sigma\left(v, X_{v}\right) d B_{v}, \quad t \in[0, T]$, in the presence of a lower solution $\alpha$ and an upper solution $\beta$ in a reverse order $(\beta \leq \alpha)$ is established. By the method of upper and lower solutions in the reverse order, it is shown that the G-SDEs have more than one solution if the drift coefficients are discontinuous functions.
\end{abstract}

Key words: Upper and lower solutions in reverse order, stochastic differential equations, G-Brownian motion, discontinuous drift coefficient, existence.

\section{INTRODUCTION}

Motivated from the risk measures, superhedging in finance and uncertainties in statistics, the G-Brownian motion was introduced by Peng (2006). The related stochastic calculus in the framework of a sublinear expectation (known as G-expectation) is developed (Peng, 2006, 2008). He introduced the stochastic differential equations driven by G-Brownian motion (GSDEs) and established the existence and uniqueness of solutions for G-SDEs with Lipschitz continuity condition on the coefficients (Peng, 2006, 2008). The G-SDEs with integral Lipschitz conditions were studied in Bai and Lin (2010) and with global Carathéodory conditions in Ren and $\mathrm{Hu}$ (2011) and Gao (2009). In contrast to the aforementioned, here the existence theory for G-SDEs whose drift coefficients are discontinuous functions is developed by the method of upper and lower solutions in the reverse order. The importance of discontinuous functions is not uncommon. For example, the unit step function or the Heaviside function $H: \mathrm{R} \rightarrow \mathrm{R}$, defined by,

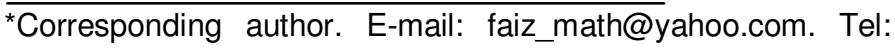
0086-18724726029.

AMS Subject Classification: $60 \mathrm{H} 10,60 \mathrm{H} 20$.
$H(x)= \begin{cases}0, & \text { if } x<0 \\ 1, & \text { if } x \geq 0\end{cases}$

is considered to be a fundamental function in engineering. It mathematically describes the switching process of voltage in an electrical circuit and arises in many discontinuous ordinary differential equations (Heikkila and Lakshmikantham, 1994). The sawtooth function or the fractional part function $\{x\}: R \rightarrow[0,1)$ is defined by

$$
\{x\}=x-\lfloor x\rfloor, \quad x \in R,
$$

where $\lfloor x\rfloor$ is the floor function (Graham et al., 1994). It has discontinuities at integers. The importance of this function is apparent from the use of sawtooth waves in music and computer graphics. The impressed voltage on a circuit could also be represented by the sawtooth function (Zill, 2009).

Furthermore, the method of upper and lower solutions is a very beneficial technique for the existence theory of boundary value problems (BVPs) for ordinary as well as partial differential equations. A vast literature is available where the lower solution $\alpha$ and upper solution $\beta$ is 
ordered as usual $\alpha \leq \beta$ (Agarwal et al., 2003; Henderson and Kunkel, 2006; Khan and Faizullah, 2009) and in the reverse order $\beta \leq \alpha$ (Cabada and Sanchez, 1996, Cabada et al., 2001; Cherpion et al., 2001). Also, Karatay et al. (2011) and Taiwo and Ogunlaran (2011) work on some BVPs. However, a very little work has been done on this method in the stochastic differential equations. In Faizullah and Piao (2011) we have established the mentioned method for backward stochastic differential equations driven by G-Brownian motion (G-BSDEs). In this paper, we introduce the upper and lower solutions method for G-SDEs in the reverse order. The following stochastic differential equation under G-Brownian motion,

$X_{t}=X_{0}+\int_{0}^{t} b\left(v, X_{v}\right) d v+\int_{0}^{t} \theta\left(v, X_{v}\right) d\langle B\rangle_{v}+\int_{0}^{t} \sigma\left(v, X_{v}\right) d B, t \in[0, T]$,

is studied. The initial condition $X_{0} \in R^{n}$ is a given constant and $\left\{\langle B\rangle_{t}\right\}_{t \geq 0}$ is the quadratic variation process of one dimensional (only for simplicity) GBrownian motion $\left\{B_{t}\right\}_{t \geq 0}$. The drift coefficient $b(t, x)$ is a discontinuous function where $\theta(t, x)$ and $\sigma(t, x)$ are Lipschitz continuous for all $x \in R^{n}$. A process $X_{t} \in M_{G}^{2}\left(0, T ; \mathrm{R}^{n}\right)$ satisfying the G-SDE is said to be its solution.

This paper is organized as follows: some basic definitions and concepts of the G-expectation are given, the method of upper and lower solutions for G-SDEs is established, the comparison theorem for G-SDEs is determined and the existence theory for G-SDEs with discontinuous drift coefficients is introduced.

\section{PRELEMINARIES}

The book (Peng, 2010) and papers (Denis et al., 2010; Peng 2008, 2009) are good references for the material here.

Let $\Omega$ be a (non-empty) basic space and $\mathrm{H}$ be a linear space of real valued functions defined on $\Omega$ such that any arbitrary constant $c \in \mathrm{H}$ and if $X \in \mathrm{H}$, then $X \mathrm{~K} \in \mathrm{H}$. We consider that $\mathrm{H}$ is the space of random variables.

\section{Definition 1}

A functional $\mathrm{E}: \mathrm{H} \rightarrow \mathrm{R}$ is said to be a sublinear expectation, if for all $X, Y \in \mathrm{H}, \quad c \in \mathrm{R}$ and $\lambda \geq 0$ it satisfies the following properties:

1. Monotonicity: if $X \geq Y$, then $\mathrm{E}[X] \geq \mathrm{E}[Y]$.
2. Constant preserving: $\mathrm{E}[c]=c$.

3. Sub-additivity: $E[X+Y] \leq E[X]+E[Y]$ or $E[X]-E[Y] \leq E[X-Y]$.

4. Positive homogeneity: $E[\lambda X]=\lambda E[X]$.

The triple $(\Omega, \mathrm{H}, \mathrm{E})$ is called a sublinear expectation space. The aforementioned functional $\mathrm{E}: \mathrm{H} \rightarrow \mathrm{R}$ is called a nonlinear expectation if it satisfies only the first two properties, that is, (1) and (2).

Consider the space of random variables $\mathrm{H}$ such that if $X_{1}, X_{2}, \ldots, X_{n} \in \mathrm{H}$, then $\varphi\left(X_{1}, X_{2}, \ldots, X_{n}\right) \in \mathrm{H}$ for each $\varphi \in \mathrm{C}_{\text {l.Lip }}\left(\mathrm{R}^{n}\right)$, where $\mathrm{C}_{\text {l.Lip }}\left(\mathrm{R}^{n}\right)$ is the space of linear functions $\varphi$ defined as the following

$C_{\text {l.Lip }}\left(R^{n}\right)=\left\{\varphi: R^{n} \rightarrow R \mid \exists C \in R^{+}, m \in N\right.$ s.t. $\left.|\varphi(x)-\varphi(y)| \leq c\left(1+|x|^{m}+|y|^{m}\right)|x-y|\right\}$,

for $x, y \in \mathrm{R}^{n}$.

\section{Definition 2}

Two n-dimensional random vectors $X$ and $\hat{X}$ defined, respectively on the sublinear expectation spaces $(\Omega, \mathrm{H}, \mathrm{E})$ and $(\hat{\Omega}, \hat{\mathrm{H}}, \hat{E})$ are said to be identically distributed, denoted by $X \sim \hat{X}$, if

$\mathrm{E}[\varphi(X)]=\hat{\mathrm{E}}[\varphi(\hat{X})], \quad \forall \varphi \in \mathrm{C}_{l . L i p}\left(\mathrm{R}^{n}\right)$.

\section{Definition 3}

Let $(\Omega, \mathrm{H}, \mathrm{E})$ be a sublinear expectation space and $X \in \mathrm{H}$ with:

$\bar{\sigma}^{2}=E\left[X^{2}\right], \underline{\sigma}^{2}=-E\left[-X^{2}\right]$.

Then $X$ is said to be G-normally distributed or $\mathbf{N}\left(0 ;\left[\bar{\sigma}^{2}, \underline{\sigma}^{2}\right]\right)$-distributed, if $\forall a, b \geq 0$ we have,

$a X+b Y \sim \sqrt{a^{2}+b^{2}} X$,

for each $Y \in \mathrm{H}$ which is independent to $X$ and $Y \sim X$.

\section{G-expectation and G-Brownian motion}

Let $\Omega=C_{0}\left(\mathrm{R}^{+}\right)$, that is, the space of all $\mathrm{R}$-valued 
continuous paths $\left(w_{t}\right)_{t \in \mathrm{R}^{+}}$with $w_{0}=0$ equipped with the norm:

$\rho\left(w^{1}, w^{2}\right)=\sum_{k=1}^{\infty} \frac{1}{2^{k}}\left(\max _{t \in[0, k]}\left|w_{t}^{1}-w_{t}^{2}\right| \wedge 1\right)$,

and consider the canonical process $B_{t}(w)=w_{t}$ for $t \in[0, \infty), w \in \Omega$, then for each fixed $T \in[0, \infty)$ we have:

$L_{p}\left(\Omega_{T}\right)=\left\{\varphi\left(B_{t_{1}}, B_{t_{2}}, \ldots B_{t_{n}}\right): t_{1}, \ldots t_{n} \in[0, T], \varphi \in \mathrm{C}_{l . L i p}\left(\mathrm{R}^{n}\right), n \in \mathrm{N}\right\}$,

where $\quad L_{i p}\left(\Omega_{t}\right) \subseteq L_{i p}\left(\Omega_{T}\right) \quad$ for $\quad t \leq T \quad$ and $L_{i p}(\Omega)=\cup_{m=1}^{\infty} L_{i p}\left(\Omega_{m}\right)$.

For $0=t_{0}<t_{1}<\ldots<t_{n}<\infty, \quad \varphi \in \mathrm{C}_{\text {l.Lip }}\left(\mathrm{R}^{n}\right)$ and each $X=\varphi\left(B_{t_{1}}-B_{t_{0}}, B_{t_{2}}-B_{t_{1}}, \ldots, B_{t_{n}}-B_{t_{n-1}}\right) \in L_{i p}(\Omega)$, $\left.\mathrm{E}\left[\varphi\left(B_{t_{1}}-B_{t_{0}}, B_{t_{2}}-B_{t_{1}}, \ldots B_{t_{n}}-B_{t_{n-1}}\right)\right]=\mathrm{E}\left[\varphi \sqrt{t_{1}-t_{0}} \xi_{1}, \ldots \sqrt{t_{n}-t_{n-1}} \xi_{n}\right)\right]$.

The conditional sublinear expectation of $X \in L_{i p}\left(\Omega_{t}\right)$ is defined by:

$\mathrm{E}\left[X \mid \Omega_{4}\right]=\mathrm{E}\left[\varphi\left(B_{t_{1}}, B_{t_{2}}-B_{t_{1}}, \ldots B_{t_{m}}-B_{t_{m-1}}\right) \mid \Omega_{4}\right]=\psi\left(B_{t_{1}}, B_{t_{2}}-B_{t_{1}}, \ldots B_{t_{j}}-B_{t_{j-1}}\right)$,

where

$\psi\left(x_{1}, \ldots, x_{j}\right)=\mathrm{E}\left[\varphi\left(x_{1}, \ldots, x_{j}, \sqrt{t_{j+1}-t_{j}} \xi_{j+1}, \ldots, \sqrt{t_{n}-t_{n-1}} \xi_{n}\right)\right]$, such that $\xi_{i}$ is G-normally distributed and $\xi_{i+1}$ is independent of $\left(\xi_{1}, \xi_{2}, \ldots, \xi_{i}\right)$ for each $i=1,2, \ldots, n-1$.

\section{Definition 4}

The sublinear expectation $\mathrm{E}: L_{i p}(\Omega) \rightarrow \mathrm{R}$ is called a G-expectation if the corresponding canonical process $\left\{B_{t}\right\}_{t \geq 0}$ on the sub-linear expectation space $\left(\Omega, L_{i p}(\Omega), \mathrm{E}\right)$ is a G-Brownian motion, that is, for $0 \leq s<t$, it satisfies the following conditions:

1. $B_{0}(w)=0$.

2. The increment $B_{t+s}-B_{t}$ is $\mathbf{N}\left(0,\left[\underline{\sigma}^{2}, \bar{\sigma}^{2}\right]\right)$ distributed and independent of $\left(B_{t_{1}}, B_{t_{2}}, \ldots, B_{t_{n}}\right)$, for each $n \in Z^{+}$and $0 \leq t_{1} \leq \ldots \leq t_{n} \leq t$.
The completion of $L_{i p}(\Omega)$ under the norm $\|X\|_{p}=\left(E\left[|X|^{p}\right]\right)^{1 / p} \quad$ for $\quad p \geq 1 \quad$ is denoted by $L_{G}^{p}(\Omega) \quad$ and $\quad L_{G}^{p}\left(\Omega_{t}\right) \subseteq L_{G}^{p}\left(\Omega_{T}\right) \subseteq L_{G}^{p}(\Omega) \quad$ for $0 \leq t \leq T<\infty$.

Note that the G-Brownian motion is not based on a particular probability space. For classical Brownian motion and probability space (Baten and Kamil, 2010).

\section{Itô's integral of G-Brownian motion}

For any $T \in \mathrm{R}^{+}$, a finite ordered subset $\pi_{T}=\left\{t_{0}, t_{1}, \ldots, t_{N}\right\}$ such that $0=t_{0}<t_{1}<\ldots<t_{N}=T$ is a partition of $[0, T] \quad$ and $\mu\left(\pi_{T}\right)=\max \left\{\left|t_{i+1}-t_{i}\right|: i=0,1, \ldots, N-1\right\}$.

A sequence of partitions of $[0, T]$ is denoted by $\pi_{T}^{N}=\left\{t_{0}^{N}, t_{1}^{N}, \ldots, t_{N}^{N}\right\}$ such that $\lim _{N \rightarrow \infty} \mu\left(\pi_{T}^{N}\right)=0$.

Consider the following simple process, let $p \geq 1$ be fixed for a given partition $\pi_{T}=\left\{t_{0}, t_{1}, \ldots, t_{N}\right\}$ of $[0, T]$,

$\eta_{t}(w)=\sum_{i=0}^{N-1} \xi_{i}(w) I_{\left[t_{i}, t_{i+1}\right)}(t)$,

where $\quad \xi_{i} \in L_{G}^{p}\left(\Omega_{t}\right), \quad i=0,1, \ldots, N-1 . \quad$ The collection containing the aforementioned type of processes, that is, containing $\eta_{t}(w)$ is denoted by $M_{G}^{p, 0}(0, T)$. The completion of $M_{G}^{p, 0}(0, T)$ under the norm $\|\eta\|=\left\{\int_{0}^{T} E\left[\left|\eta_{v}\right|^{p}\right] d v\right\}^{1 / p}$ is denoted by $M_{G}^{p}(0, T)$ and for $1 \leq p \leq q, \quad M_{G}^{p}(0, T) \supset M_{G}^{q}(0, T)$.

\section{Definition 5}

For each $\eta_{t} \in M_{G}^{2,0}(0, T)$, the Itô's integral of GBrownian motion is defined by:

$$
I(\eta)=\int_{0}^{T} \eta_{v} d B_{v}=\sum_{i=0}^{N-1} \xi_{i}\left(B_{t_{i+1}}-B_{t_{i}}\right) .
$$

\section{Definition 6}

An increasing continuous process $\quad\left\{\langle B\rangle_{t}\right\}_{t \geq 0}$ with $\langle B\rangle_{0}=0$, defined by: 
$\langle B\rangle_{t}=\lim _{N \rightarrow \infty} \sum_{i=0}^{N-1}\left(B_{t_{i+1}^{N}}-B_{t_{i}^{N}}\right)^{2}=B_{t}^{2}-2 \int_{0}^{t} B_{v} d B_{v}$,

is called the quadratic variation process of G-Brownian motion. For the details of the following two definitions is in the work of Denis et al. (2010).

\section{Definition 7}

Let $\mathrm{B}(\Omega)$ be the Borel $\sigma$-algebra of $\Omega$ and $\mathrm{P}$ be a (weakly compact) collection of probability measures $P$ defined on $(\Omega, \mathrm{B}(\Omega))$, then the capacity $\hat{c}($.) associated to $\mathbf{P}$ is defined by:

$\hat{c}(A)=\sup _{P \in \mathrm{P}} P(A), \quad A \in \mathrm{B}(\Omega)$.

\section{Definition 8}

A set $A$ is said to be polar if its capacity is zero, that is, $\hat{c}(A)=0$ and a property holds "quasi-surely" (q.s.) if it holds outside a polar set. Throughout this paper for $X=\left(x_{1}, x_{2}, \ldots, x_{n}\right), Y=\left(y_{1}, y_{2}, \ldots, y_{n}\right), \quad X \leq Y$ means $x_{i} \leq y_{i}, \quad i=1,2, \ldots, n$.

\section{THE METHOD OF UPPER AND LOWER SOLUTIONS}

Recall (Assing and Manthey, 1995; Halidias and Michta, 2008; Halidias and Kloeden; 2006; Ladde and Lakshmikantham, 1980) for the concept of upper and lower solutions in the sense of classical SDEs.

\section{Definition 9}

A process $\beta_{t} \in M_{G}^{2}\left(0, T ; \mathrm{R}^{n}\right)$ is said to be an upper solution of the G-SDE on the interval $[0, T]$ if the inequality (interpreted component wise):

$\beta_{t} \leq \beta_{s}+\int_{s}^{t} b\left(v, \beta_{v}\right) d v+\int_{s}^{t} \theta\left(v, \beta_{v}\right) d\langle B\rangle_{v}+\int_{s}^{t} \sigma\left(v, \beta_{v}\right) d B_{v}, 0 \leq s \leq t \leq T$,

holds quasi-surely (q.s.)

\section{Definition 10}

A process $\alpha_{t} \in M_{G}^{2}\left(0, T ; \mathrm{R}^{n}\right)$ is said to be a lower solution of the G-SDE on the interval $[0, T]$ if the inequality (interpreted component wise):

$$
\alpha_{l} \geq \alpha_{s}+\int_{s}^{t} b\left(v, \alpha_{v}\right) d v+\int_{s}^{t} \theta\left(v, \alpha_{v}\right) d\langle B\rangle_{v}+\int_{s}^{t} \sigma\left(v, \alpha_{v}\right) d B_{v}, 0 \leq s \leq t \leq T,
$$

holds q.s.

Assume that $\alpha_{t}$ and $\beta_{t}$ are the respective lower and upper solutions of the G-SDE:

$d X_{t}=b(t, w) d t+\theta\left(t, X_{t}\right) d<B>_{t}+\sigma\left(t, X_{t}\right) d B_{t}, \quad t \in[0, T]$.

Define two functions $p, r:[0, T] \times \mathrm{R}^{d} \times \Omega \rightarrow \mathrm{R}^{d}$ by:

$$
\begin{aligned}
& p(t, x, w)=\min \left\{\alpha_{t}(w), \max \left\{\beta_{t}(w), x\right\}\right\} \\
& r(t, x, w)=p(t, x, w)-x,
\end{aligned}
$$

and consider the stochastic differential equation:

$$
d X_{t}=\tilde{b}(t, w) d t+\tilde{\theta}\left(t, X_{t}\right) d<B>_{t}+\sigma\left(t, X_{t}\right) d B_{t}, \quad t \in[0, T]
$$

with a given initial condition $X_{0}$, where

$\tilde{b}(t, x, w)=b(t, w)+r(t, x, w)$,

$\tilde{\theta}(t, x, w)=\theta(p(t, x, w))$,

$\tilde{\sigma}(t, x, w)=\sigma(p(t, x, w))$,

are Lipschitz continuous. The G-SDE Equation 4 has a unique solution $X_{t} \in M_{G}^{2}\left(0, T ; \mathrm{R}^{n}\right)$ (Gao, 2010; Peng, 2006, 2008).

Remind (Li and Peng, 2011; Nutz and Soner, 2011; Song, 2011) for stopping times in G-expectation. For the aforementioned processes $X_{t}$ and $\alpha_{t}$ with the same initial values and $a \in Q^{*} \cap[0, T)$, define the following stopping times:

$\left\{\begin{array}{c}\tau^{a}=\inf \left\{t \geq a: X_{t} \leq \alpha_{t}\right\}, \\ \tau_{1}^{a}=\inf \left\{t>\tau^{a}: X_{t}>\alpha_{t}\right\}\end{array}\right.$

q.s.

Similarly, for the processes $X_{t}$ and $U_{t}$ with the same initial values and $a \in Q^{*} \cap[0, T)$, define the following stopping times:

$$
\left\{\begin{array}{c}
\tau^{a}=\inf \left\{t \geq a: X_{t} \geq \beta_{t}\right\}, \\
\tau_{1}^{a}=\inf \left\{t>\tau^{a}: X_{t}<\beta_{t}\right\},
\end{array}\right.
$$


q.s.

Moreover, it is obvious to see that

$\left\{\tau^{a}: a \in Q^{*} \cap[0, T)\right\} \cup\left\{\tau_{1}^{a}: a \in Q^{*} \cap[0, T)\right\}=[0, T]$,

where $Q^{*}$ is the set of non-negative rational numbers.

\section{COMPARISON THEOREM FOR G-SDES}

The following lemma will be used in the next comparison theorem.

\section{Lemma 1}

Assume that the respective lower and upper solutions $\alpha_{t}$ and $\beta_{t}$ of the G-SDE Equation 2 satisfy the condition $\alpha_{t} \geq \beta_{t}$ for $t \in[0, T]$ q.s. Then $\alpha_{t}$ and $\beta_{t}$ are lower and upper solutions of the G-SDE Equation 4, respectively.

\section{Proof}

Let $\beta_{t}$ be an upper solution of the G-SDE Equation 2. As $\beta_{t} \leq \alpha_{t}$ for $t \in[0, T]$. Thus, $p\left(t, \beta_{t}\right)=\beta_{t}$ and $r\left(t, \beta_{t}\right)=0$, therefore we have:

$\beta_{s}+\int_{s}^{t} \tilde{b}\left(v, \beta_{v}\right) d v+\int_{s}^{t} \tilde{\theta}\left(v, \beta_{v}\right) d\langle B\rangle_{v}+\int_{s}^{t} \tilde{\sigma}\left(v, \beta_{v}\right) d B_{v}$

$=\beta_{s}+\int_{s}^{t}\left[b(v, w)+r\left(v, \beta_{v}\right)\right] d v$

$+\int_{s}^{t} \theta\left(v, p\left(v, \beta_{v}\right)\right) d\langle B\rangle_{v}+\int_{s}^{t} \sigma\left(v, p\left(v, \beta_{v}\right)\right) d B_{v}$

$=\beta_{s}+\int_{s}^{t} b(v, w) d v+\int_{s}^{t} \theta\left(v, \beta_{v}\right) d\langle B\rangle_{v}+\int_{s}^{t} \sigma\left(v, \beta_{v}\right) d B_{v} \geq \beta_{t}$.

Hence, $\beta_{t}$ is an upper solution of Equation 4. Also, $\beta_{t} \leq \alpha_{t}$ for $t \in[0, T]$ yields $p\left(t, \alpha_{t}\right)=\alpha_{t} \quad$ and $r\left(t, \alpha_{q}\right)=0$. Thus,

$\alpha_{s}+\int_{s}^{t} \tilde{b}\left(v, \alpha_{v}\right) d v+\int_{s}^{t} \tilde{\theta}\left(v, \alpha_{v}\right) d\langle B\rangle_{v}+\int_{s}^{t} \tilde{\sigma}\left(v, \alpha_{v}\right) d B_{v}$

$=\alpha_{s}+\int_{s}^{t}\left[b(v, w)+r\left(v, \alpha_{v}\right)\right] d v$

$+\int_{s}^{t} \theta\left(v, p\left(v, \alpha_{v}\right)\right) d\langle B\rangle_{v}+\int_{s}^{t} \sigma\left(v, p\left(v, \alpha_{v}\right)\right) d B_{v}$

$=\alpha_{s}+\int_{s}^{t} b(v, w) d v+\int_{s}^{t} \theta\left(v, \alpha_{v}\right) d\langle B\rangle_{v}+\int_{s}^{t} \sigma\left(v, \alpha_{v}\right) d B_{v} \leq \alpha_{t}$.

Hence, $\alpha_{t}$ is a lower solution of Equation 4.

\section{Theorem 1}

Assume that:

1. The function $b$ is measurable with $\int_{0}^{t} E\left[|b(v, .)|^{2}\right] d v<\infty$, where $\theta(t, x) \quad$ and $\sigma(t, x) \quad$ are Lipschitz continuous in $x$.

2. The respective lower and upper solutions $\alpha_{t}$ and $\beta_{t}$ of the G-SDE Equation 2 with $\mathrm{E}\left[\left|\alpha_{t}\right|^{2}\right]<\infty, \quad \mathrm{E}\left[\left|\beta_{t}\right|^{2}\right]<\infty$ satisfy $\alpha_{t} \geq \beta_{t}$ for $t \in[0, T]$.

3. Also, $X_{0} \in \mathrm{R}^{n}$ is a given initial value with $\mathrm{E}\left[\left|X_{0}\right|^{2}\right]<\infty$ and $\beta_{0} \leq X_{0} \leq \alpha_{0}$.

Then, there exists a unique solution $X_{t} \in M_{G}^{2}\left(0, T ; \mathrm{R}^{n}\right)$ of the G-SDE Equation 2 such that $\beta_{t} \leq X_{t} \leq \alpha_{t}$ for $t \in[0, T]$ q.s.

\section{Proof}

Define the functions $p, r:[0, T] \times \mathrm{R}^{d} \times \Omega \rightarrow \mathrm{R}^{d} \quad$ by Equation 3 and consider the G-SDE Equation 4.

Now, the G-SDE Equation 4 has a unique solution and by lemma 1 if $\alpha_{t}$ and $\beta_{t}$ are lower and upper solutions of the G-SDE Equation 2, respectively, then they are the respective lower and upper solutions for the G-SDE Equation 4. Moreover, it is easy to note that any solution $X_{t}$ of the modified G-SDE Equation 4, such that:

$\beta_{t} \leq X_{t} \leq \alpha_{t}, t \in[0, T]$

q.s. is also a solution of the G-SDE Equation 2. Hence, we only need to show that any solution $X_{t}$ of the problem of Equation 4 satisfies the inequality of Equation 7. Assume that there exists an interval $\left(\tau_{1}, \tau_{2}\right) \subset[0, T]$, such that:

$$
\left\{\begin{array}{c}
X_{\tau_{1}}=\beta_{\tau_{1}}, \\
X_{t}<\beta_{t}, \quad t \in\left(\tau_{1}, \tau_{2}\right),
\end{array}\right.
$$

where $\tau_{1}$ and $\tau_{2}$ are arbitrary stopping times. Then,

$$
\begin{aligned}
X_{t}-\beta_{t} & =\int_{\tau_{1}}^{t} \tilde{b}\left(v, X_{v}\right) d v+\int_{\tau_{1}}^{t} \tilde{\theta}\left(v, X_{v}\right) d\langle B\rangle_{v}+\int_{\tau_{1}}^{t} \tilde{\sigma}\left(v, X_{v}\right) d B_{v} \\
& -\int_{\tau_{1}}^{t} \tilde{b}\left(v, \beta_{v}\right) d v-\int_{\tau_{1}}^{t} \tilde{\theta}\left(v, \beta_{v}\right) d\langle B\rangle_{v}-\int_{\tau_{1}}^{t} \tilde{\sigma}\left(v, \beta_{v}\right) d B_{v} \\
& =\int_{\tau_{1}}^{t}\left[b(v, w)+r\left(v, X_{v}\right)\right] d v+\int_{\tau_{1}}^{t} \theta\left(v, p\left(v, X_{v}\right)\right) d\langle B\rangle_{v} \\
& +\int_{\tau_{1}}^{t} \sigma\left(v, p\left(v, X_{v}\right)\right) d B_{v}-\int_{\tau_{1}}^{t}\left[b(v, w)+r\left(v, \beta_{v}\right)\right] d v \\
& -\int_{\tau_{1}}^{t} \theta\left(v, p\left(v, \beta_{v}\right)\right) d\langle B\rangle_{v}-\int_{\tau_{1}}^{t} \sigma\left(v, p\left(v, \beta_{v}\right)\right) d B_{v} .
\end{aligned}
$$


Since $\beta_{t} \leq \alpha_{t}$ gives $p\left(t, \beta_{t}\right)=\beta_{t}$ and $r\left(t, \beta_{t}\right)=0$, and $X_{t}<\beta_{t}$ yields $p\left(t, X_{t}\right)=\beta_{t}$. Thus,

$$
\begin{aligned}
X_{t}-\beta_{t} & =\int_{\tau_{1}}^{t} r\left(v, X_{v}\right) d v+\int_{\tau_{1}}^{t} \theta\left(v, \beta_{v}\right) d\langle B\rangle_{v}+\int_{\tau_{1}}^{t} \sigma\left(v, \beta_{v}\right) d B_{v} \\
& -\int_{\tau_{1}}^{t} r\left(v, \beta_{v}\right) d v-\int_{\tau_{1}}^{t} \theta\left(v, \beta_{v}\right) d\langle B\rangle_{v}-\int_{\tau_{1}}^{t} \sigma\left(v, \beta_{v}\right) d B_{v} \\
& =\int_{\tau_{1}}^{t} r\left(v, X_{v}\right) d v .
\end{aligned}
$$

But $\quad X_{t}<\beta_{t} \quad$ for $\quad t \in\left(\tau_{1}, \tau_{2}\right) \quad$ implies $r\left(t, X_{t}\right)=\beta_{t}-X_{t}>0$. Hence,

$X_{t}-\beta_{t}=\int_{\tau_{1}}^{t} r\left(v, X_{v}\right) d v>0$

this is a contradiction. Hence, $X_{t} \geq \beta_{t}$ for $t \in[0, T]$.

Now we assume that there exists an arbitrary interval $\left(\tau_{1}, \tau_{2}\right) \subset[0, T]$, such that:

$$
\left\{\begin{array}{l}
X_{\tau_{1}}=\alpha_{\tau_{1}}, \\
X_{t}>\alpha_{t}, \quad t \in\left(\tau_{1}, \tau_{2}\right) .
\end{array}\right.
$$

Then

$$
\begin{aligned}
& \alpha-X_{t}=\int_{\tau_{1}}^{t} \tilde{b}(v, \alpha) d v+\int_{\tau_{1}}^{t} \tilde{\theta}(v, \alpha) d\langle B\rangle_{v}+\int_{\tau_{1}}^{\tau} \tilde{\alpha}(v, \alpha) d B_{v} \\
& \int_{\tau_{1}}^{t} \tilde{b}\left(v, X_{v}\right) d v-\int_{\tau}^{t} \tilde{\alpha}\left(v, X_{v}\right) d\langle B\rangle_{v}-\int_{\tau}^{t} \tilde{\alpha}\left(v, X_{v}\right) d B_{v} \\
& =\int_{\tau_{1}}^{t}[b(v, w)+r(v, \alpha)) d v+\int_{\tau_{1}}^{r} \theta(v, p(v, \alpha)) d\langle B\rangle_{v}+\int_{\tau_{1}}^{t} \alpha(v, p(v, \alpha)) d B_{v} \\
& \int_{\tau_{1}}^{t}\left[b(v, w)+r\left(v, X_{v}\right)\right] d v-\int_{\tau_{1}}^{t} \theta\left(v, p\left(v, X_{v}\right)\right) d\langle B\rangle_{v}-\int_{\tau_{1}}^{t} \sigma\left(v, p\left(v, X_{v}\right)\right) d B \text {. }
\end{aligned}
$$

Similar argument as the aforementioned yields:

$$
\alpha_{t}-X_{t}=-\int_{\tau_{1}}^{t} r\left(v, X_{v}\right) d v>0
$$

because $r\left(t, X_{t}\right)<0$, which is again a contradiction. Hence, $X_{t} \leq \alpha_{t}$ for $t \in[0, T]$.

\section{G-SDES WITH DISCONTINUOUS COEFFICIENTS}

DRIFT

Now we consider the following G-SDE:

$d X_{t}=b\left(t, X_{t}\right) d t+\theta\left(t, X_{t}\right) d\langle B\rangle_{t}+\sigma\left(t, X_{t}\right) d B_{t}, \quad t \in[0, T], \quad$ (8) where the drift coefficient does not need to be continuous. But suppose that it is increasing, that is, if $x \geq y$, then $b(t, x) \geq b(t, y)$ (where the inequalities are interpreted component wise) and $\theta(t, x), \quad \sigma(t, x)$ are Lipschitz continuous in $x$.

For the following definition and theorem, the work of Heikkila and Hu (1993) has the details.

\section{Definition 11}

An ordered metric space $M$ is called regularly (resp. fully regularly) ordered, if each monotone and order (resp. metrically) bounded ordinary sequence of $M$ converges.

\section{Theorem 2}

If $[a, b]$ is a nonempty order interval in a regularly ordered metric space, then each increasing mapping $f:[a, b] \rightarrow[a, b]$ has the least and the greatest fixed point.

\section{Theorem 3}

Assume that:

1. The function $b(t, x)$ is increasing in $x$ where $\theta(t, x)$ and $\sigma(t, x)$ are Lipschitz continuous in $x$.

2. $\alpha_{t}$ and $\beta_{t}$ are lower and upper solutions of the GSDE of Equation 8 with $\int_{0}^{t} \mathrm{E}\left[\left|b\left(\alpha_{v}\right)\right|^{2}\right] d v<\infty$, $\int_{0}^{t} \mathrm{E}\left[\left|b\left(\beta_{v}\right)\right|^{2}\right] d v<\infty$, respectively and $\beta_{t} \leq \alpha_{t}$ for $t \in[0, T]$.

Then, there exists at least one solution $X_{t} \in M_{G}^{2}\left(0, T ; \mathrm{R}^{n}\right)$ of the G-SDE Equation 8 , such that $\beta_{t} \leq X_{t} \leq \alpha_{t}$ for $t \in[0, T]$ q.s.

\section{Proof}

Denote the space of all $d$-dimensional stochastic processes by $\mathbf{H}$, that is, $\mathrm{H}=\left\{X=\left\{X_{t}, t \in[0, T]\right\}: \mathrm{E}\left[\left|X_{t}\right|^{2}\right]<\infty\right\}$ with the norm $\|\eta\|=\left\{\int_{0}^{T} E\left[\left|\eta_{v}\right|^{2}\right] d v\right\}^{1 / 2}$ for all $t \in[0, T]$, which is a Banach space (Peng, 2006, 2008, 2010). Also see the work of Xu et al., (2011) for Banach spaces.

Now represent the order interval $\left[\beta_{t}, \alpha_{t}\right]$ in $\mathrm{H}$ by $\mathrm{K}$, 
that is, $\mathbf{K}=\left\{X_{t}: X_{t} \in \mathrm{H}\right.$ and $\left.\beta_{t} \leq X_{t} \leq \alpha_{t}\right\}$ for $t \in[0, T]$, which is closed and bounded by the aforementioned norm. By using the monotone convergence theorem (Denis et al., 2010), one can prove the convergence of a monotone sequence that belongs to $\mathbf{K}$ in $\mathbf{H}$. Thus, $\mathbf{K}$ is a regularly ordered metric space with the aforementioned norm. It is clear that for any process $U_{t} \in \mathbf{K}, \alpha_{t}$ and $\beta_{t}$ are lower and upper solutions for the G-SDE

$d X_{t}=b\left(t, U_{t}\right) d t+\theta\left(t, X_{t}\right) d\langle B\rangle_{t}+\sigma\left(t, X_{t}\right) d B_{t}, \quad t \in[0, T]$. (9)

Thus, by Theorem 1, for any given initial value $X_{0} \in \mathrm{R}^{n}$ with $E\left[\left|X_{0}\right|^{2}\right]<\infty$ and $\beta_{0} \leq X_{0} \leq \alpha_{0}$, the GSDE Equation 9 has a unique solution $X_{t} \in M_{G}^{2}\left(0, T ; R^{n}\right)$ such that $\beta_{t} \leq X_{t} \leq \alpha_{t}$ for $t \in[0, T]$ q.s.

Define an operator $f: \mathbf{K} \rightarrow \mathbf{K}$ by $f\left(U_{t}\right)=X_{t}$, where $X_{t}$ is the unique solution of the G-SDE equation 9. We will use Theorem 2 to show that $f$ has a fixed point, which is then the required solution. If we show that $f$ is an increasing mapping, then it has a fixed point.

We have to prove that if $U_{t}^{(1)}$ and $U_{t}^{(2)}$ are stochastic processes in $\mathrm{K}$ such that $U_{t}^{(1)} \leq U_{t}^{(2)}$ then $X_{t}^{(1)} \leq X_{t}^{(2)}$ for all $t \in[0, T]$, where $X_{t}^{(1)}=f\left(U_{t}^{(1)}\right) \quad$ and $X_{t}^{(2)}=f\left(U_{t}^{(2)}\right)$.

Let $U_{t}^{(1)} \leq U_{t}^{(2)} \quad$ for all $t \in[0, T]$ and define $X_{t}^{(1)}=f\left(U_{t}^{(1)}\right), \quad X_{t}^{(2)}=f\left(U_{t}^{(2)}\right), \quad$ where $\quad U_{t}^{(1)}, U_{t}^{(2)} \in \mathbf{K}$. Since the drift coefficient $b$ is an increasing function, therefore $X_{t}^{(1)}$ is an upper solution of the G-SDE:

$$
X_{t}=X_{0}+\int_{0} b\left(v, U_{v}^{22}\right) d v+\int_{0} a\left(v, X_{v}\right) d(B\rangle_{v}+\int_{0} \alpha\left(v, X_{v}\right) d B, t \in[0, T]
$$

But this problem has a lower solution $\alpha_{t}$. Hence, by Theorem 1, the G-SDE Equation 10 has a solution $X_{t}^{(2)}$ such that $X_{t}^{(1)} \leq X_{t}^{(2)} \leq \alpha_{t}$. Thus, $f$ is an increasing mapping and by theorem 2, it has a fixed point $X_{t}^{(*)}=f\left(X_{t}^{(*)}\right) \in \mathbf{K}$, such that $\alpha_{t} \leq X_{t}^{(*)} \leq \beta_{t}$ q.s. where

$$
X_{t}^{(*)}=X_{0}+\int_{0}^{t} b\left(v, X_{v}^{(*)}\right) d v+\int_{0}^{t} \theta\left(v, X_{v}^{(*)}\right) d\langle B\rangle_{v}+\int_{0}^{t} \sigma\left(v, X_{v}^{(*)}\right) d B_{v} .
$$

\section{Example}

Consider the following scalar G-SDE: $d X_{t}=H\left(X_{t}\right) d t+d\langle B\rangle_{t}+d B_{t}, \quad t \in[0, T]$.

Since the Heaviside function $H: \mathrm{R} \rightarrow \mathrm{R}$ is an increasing function. Hence, $\beta_{t}=\beta_{0}+\int_{0}^{t} d\langle B\rangle_{v}+\int_{0}^{t} d B_{v}$ and $\alpha_{t}=\alpha_{0}+\int_{0}^{t} d v+\int_{0}^{t} d\langle B\rangle_{v}+\int_{0}^{t} d B_{v}$ are the respective upper and lower solutions of the scalar G-SDE Equation 11, which are shown as follows:

$$
\begin{aligned}
\beta_{t} & =\beta_{0}+\int_{0}^{t} d\langle B\rangle_{v}+\int_{0}^{t} d B_{v} \\
& =\beta_{s}+\int_{s}^{t} d\langle B\rangle_{v}+\int_{s}^{t} d B_{v} \\
& \leq \beta_{s}+\int_{s}^{t} H\left(\beta_{v}\right) d v+\int_{s}^{t} d\langle B\rangle_{v}+\int_{s}^{t} d B_{v},
\end{aligned}
$$

where $\beta_{s}=\beta_{0}+\int_{0}^{s} d\langle B\rangle_{v}+\int_{0}^{s} d B_{v}$ for each fixed $s$ such that $0 \leq s \leq t \leq T$. Hence, $\quad \beta_{t}=\beta_{0}+\int_{0}^{t} d\langle B\rangle_{v}+\int_{0}^{t} d B_{v}$ is an upper solution.

Similarly,

$$
\begin{aligned}
\alpha_{t} & =\alpha_{0}+\int_{0}^{t} d v+\int_{0}^{t} d\langle B\rangle_{v}+\int_{0}^{t} d B_{v} \\
& =\alpha_{s}+\int_{s}^{t} d v+\int_{s}^{t} d\langle B\rangle_{v}+\int_{s}^{t} d B_{v} \\
& \geq \alpha_{s}+\int_{s}^{t} H\left(\alpha_{v}\right) d v+\int_{0}^{t} d\langle B\rangle_{v}+\int_{s}^{t} d B_{v},
\end{aligned}
$$

where $\alpha_{s}=\alpha_{0}+\int_{0}^{s} d v+\int_{0}^{s} d\langle B\rangle_{v}+\int_{0}^{s} d B_{v}$ for each fixed $s$ such that $0 \leq s \leq t \leq T$. Thus, $\alpha_{t}=\alpha_{0}+\int_{0}^{t} d v+\int_{0}^{t} d\langle B\rangle_{v}+\int_{0}^{t} d B_{v} \quad$ is the required lower solution. Hence, by the aforementioned theorem the GSDE Equation 11 has at least one solution $X_{t}^{(*)}$ such that $\beta_{t} \leq X_{t}^{(*)} \leq \alpha_{t}$ for $t \in[0, T]$.

\section{REFERENCES}

Agarwal RP, Thompson HB, Tisdell CC (2003). Three-point boundary value problems for second-order discrete equations. Comput. Math. Appl., pp. 1-7.

Assing S, Manthey R (1995). The behaviour of solutions of stochastic differential inequalities. Probab. Theory Rel., 103: 493-514.

Bai $X$, Lin Y (2010). On the existence and uniqueness of solutions to stochastic differential equations driven by G-Brownian motion with integral-lipschitz coefficients. arXiv:math. PR/1002.1046v2.

Baten MdA, Kamil AA (2010). Direct solution of Riccati equation arising in inventory production control in a Stochastic manufacturing system. Int. J. Phys. Sci., 5(7): 931-934.

Cabada A, Habets P, Lois S (2001). Monotone method of the Neumann problem with lower and upper solutions in the reverse order. Appl. Math. Comput., 117: 1-4.

Cabada A, Sanchez L (1996). A positive operator approach to the Neumann problem for second order ordinary differential equation. $\mathrm{J}$. Math. Anal. Appl., 204: 774-785.

Cherpion M, Coster CD, Habets P (2001). A constructive monotone iterative method for second order BVP in the presence of lower and 
upper solutions. Appl. Math. Comput., 123: 75-91.

Denis L, Hu M, Peng S (2010). Function spaces and capacity related to a sublinear expectation: Application to G-Brownian motion paths. Potential Anal., 34: 139-161.

Faizullah, Piao D (2011). A note on the existence of solutions for bachward stochastic differential equations under G-Brownian motion. World Appl. Sci. J. (to be appeared)

Gao F (2009). Pathwise properties and homeomorphic flows for stochastic differential equations driven by G-Brownian motion. Stoch. Proc. Appl., 10: 3356-3382.

Graham RL, Knuth DE, Patashnik O (1994). Concrete Mathematics. Addison-wesley Publishing Company, Second Edition.

Halidias N, Michta M (2008). The method of upper and lower solutions of stochastic differential equations and applications. Stoch. Anal. Appl., 26: 16-28.

Halidias N, Kloeden PE (2006). A note on strong solutions of stochastic differential equations with a discontinuous drift coefficient. J. Appl. Math. Stoch. Anal., DOI 10.1155/JAMSA/2006/73257, pp. 1-6.

Heikkila S, Hu S (1993). On fixed points of multifunctions in ordered spaces. Appl. Anal., 51: 115-127.

Heikkila S, Lakshmikantham V (1994). Monotone Iterative Techniques for Discontinuous Nonlinear Differential Equations, Monographs and Textbooks in Pure and Applied Mathematics. Marcel Dekker, New York, p. 181.

Henderson J, Kunkel CJ (2006). Singular discrete higher order boundary value problems. Int. J. Difference Equ., 1: 119-133.

Karatay I, Bayramoglu SR, Yildiz B, Kokluce B (2011). Matrixed stability of the difference schemes for nonlocal boundary value problems for parabolic differential equations. Int. J. Phys. Sci., 6(4): 819-827.

Khan RA, Faizullah RM (2009). Existence and approximation of solutions of boundary value problems on time scales. Adv. Dyn. Syst. Appl., 4: 197-209.

Ladde GS, Lakshmikantham V (1980). Random Differential Inequalities. Mathematics in Science and Engineering, Acadmic Press, New York, p. 150.

Li X, Peng S (2011). Stopping times and related Ito's calculus with GBrowniain motion. Stoch. Proc. Appl., doi: 10.1016/j.spa. 2011.03.009.

Nutz M, Soner HM (2011). Superhedging and dynamic risk measures under volatility uncertainty. arXiv:math.PR/:1011.2958v1.

Peng S (2006). G-expectation, G-Brownian motion and related stochastic calculus of Ito's type. The Abel Symposium 2005, Abel Symposia 2, Edit. Benth Springer-Vertag, pp. 541-567.
Peng S (2008). Multi-dimentional G-Brownian motion and related stochastic calculus under G-expectation. Stoch. Proc. Appl., 12: 2223-2253.

Peng S (2010). Nonlinear expectations and stochastic calculus under uncertainty. First Edition, arXiv:math. PR/:1002.4546v1.

Peng S (2009). Survey on normal distributions, central limit theorem, GBrownian motion and the related stochastic calculus under sublinear expectations. Sci. China Ser. A., 7: 1391-1411.

Ren $Y, H u L$ (2011). A note on the stochastic differential equations driven by G-Brownian motion. Stat. Probabil. Lett., 81: 580-585.

Song $Y$ (2011). Properties of hitting times for G-martingale and their applications. Stoch. Proc. Appl., doi: 10.1016/j.spa. 2011.04.007.

Taiwo OA, Ogunlaran OM (2011). A non-polynomial spline method for solving linear fourth-order boundary-value problems. Int. J. Phys. Sci., 6(13): 3246-3254.

Xu TZ, Rassias JM, Xu WX (2011). A fixed point approached to the stability of a general mixed additive-cubic functional equation in quasi fuzzy normed spaces. Int. J. Phys. Sci., 6(2): 313-324.

Zill DG (2009). A First Course in Differential Equations with Modeling Applications. Brooks/Cole Cengage Learning, Ninth Edition. 